\title{
Short-Length Raptor Codes for Mobile Free-Space Optical Channels
}

\author{
Wenzhe Zhang and Steve Hranilovic \\ Dept. Electrical and Computer Engineering, McMaster University \\ Hamilton, ON, Canada \\ zhangwe@grads.ece.mcmaster.ca, hranilovic@mcmaster.ca
}

\begin{abstract}
Free-space optical (FSO) links are competitive wireless links offering high data rate, security and low system complexity. For mobile applications, e.g., from a ground base station to an unmanned aerial vehicle (UAV), the FSO channel can suffer from severe instantaneous misalignment. This time varying misalignment is unknown to the transmitter and causes data packet corruption and erasure. As a result, the application of traditional fixed-rate erasure coding techniques is difficult. In this paper, we consider the application of rateless Raptor codes for such mobile FSO channels. Due to the high data rates required, short-length (16-1024) Raptor codes are designed and simulated on a severe jitter FSO channel. A key advantage of Raptor codes is their independence on channel state, no matter how large the misalignment. With a 1 Gbps transmitter, the designed Raptor code with $k=64$ message packets offers $560 \mathrm{Mbps}$ data rate and decoding cost of 4.14 operations per packet when transmitting power is $20 \mathrm{dBm}$. In contrast, a traditional automatic repeatrequest (ARQ) algorithm technique on the same FSO jitter channel achieves a rate of $60 \mathrm{Mbps}$.
\end{abstract}

\section{INTRODUCTION}

Free-space optical (FSO) links provide line-of-sight (LOS) data links by modulating the instantaneous intensity of a laser and allowing it to propagate through the atmosphere to a receiver. Such links are relatively inexpensive and provide high data rates free of jamming and interference. Most commercial FSO links operate at $1-2$ Gbps over ranges of $1-3 \mathrm{~km}$, however, links of up to $80 \mathrm{Gbps}$ over $3.4 \mathrm{~km}$ have been demonstrated [1]. Although most FSO links are stationary, they have also been considered for mobile applications. Applications such as ship-to-ship [2], ground-to-air and airto-air FSO communication systems have been studied [3], [4]. FSO links satisfy the needs of long-range deep-space communications [6] and have also been considered for military wireless communications [7].

In mobile FSO channels, the transmitter must track the motion of the receiver in order to maintain a LOS link. Consider the ground-to-unmanned aerial vehicle (UAV) FSO link in Fig.1 where the speed of the UAV can reach several hundred meters per second [9]. In this scenario, tracking is typically accomplished by mechanical components such as a 2-dimensional rotating gimbal which is oriented based on fed back global positioning system (GPS) data from the UAV [3], [4]. Tracking is made more difficult due to the use of FSO transmitters with a small divergence angle to minimize geometric loss. A dramatic variation in the received SNR is caused by misalignment of the transmitted beam and the

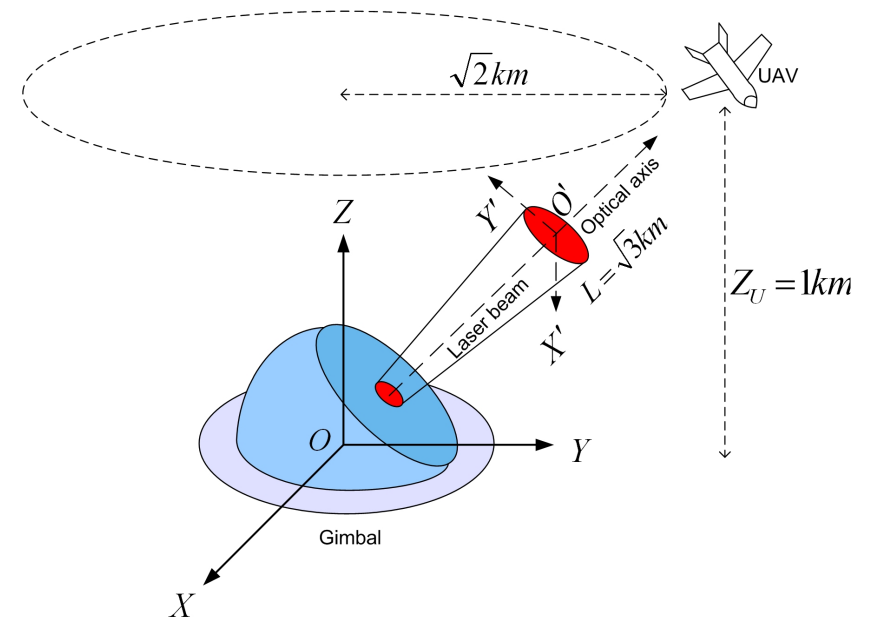

Fig. 1. Ground-to-UAV Mobile FSO channel configuration

detector due to mechanical pointing uncertainty error and GPS positioning error. The total tracking error is the combination of these two errors and limits the data rates of such links.

For these severe jitter channels, the total tracking error is unknown to the transmitter and changes slowly in time with respect to the bit period. In order to improve the data rates of such links, coding techniques can be considered. Fixed-rate coding techniques are not appropriate for such links due to the lack of channel state information and the slow variation of the channel. As a result, in this work we consider rateless erasure correcting codes called Raptor codes [5]. In these codes, the transmitter creates a stream of output packets based on the message to be transmitted. The receiver collects these packets and can, with high probability, decode the entire message once enough packets are received.

In this work, we consider for the first time the application of Raptor codes to mobile FSO links to overcome the severe limitations imposed due to misalignment errors. In Sec. II, a channel model for the ground-to-UAV channel is presented including the combined effects of atmospheric scintillation and misalignment error. In Sec. III, a novel set of short-length (16-1024) Raptor codes are designed with high rate and low decoding cost. Previous Raptor code designs consider lengths of at least 9500 [10] which require complex decoders and incur a large latency making them impractical for high-speed FSO channels. Sec. IV presents detailed numerical simulations of 
the designed system and the paper concludes in Sec. V.

\section{FSO CHANNEL MODEL}

\section{A. Channel description}

For the ground-to-UAV system in Fig. 1, a practical tracking system has been developed which includes a gimbal for rotating the laser transmitter in two dimensions and GPS for UAV position information [3]. In this paper, we use the experimentally measured properties of this tracking system and include the effects of atmospheric scintillation [3], [11].

As mentioned in [3], the gimbal is at the origin, $O$, of coordinate system $X Y Z$ in Fig. 1. A UAV flies in a $\sqrt{2} \mathrm{~km}$ circle at a speed of $100 \mathrm{~m} / \mathrm{s}$ with altitude $Z_{U}=1 \mathrm{~km}$, and the circle is parallel to plane $X Y$. The ground-to-UAV distance along the optical axis is $L=\sqrt{3} \mathrm{~km}$. Define the plane $X^{\prime} Y^{\prime}$ to be transverse to the optical axis. It is assumed, as in [3], that initial acquisition between UAV and base station has been completed before tracking. As in [3], the tracking process is performed periodically by updating the UAV position by GPS at a tracking interval $T_{t r}=50 \mathrm{~ms}$ and orienting the gimbal accordingly.

Define $\mathcal{X}$ as the transmitted optical intensity signal, $\mathcal{Y}$ as received signal and $n$ as additive Gaussian noise at receiver with standard deviation $\sigma_{n}$. The channel can be modelled as [8]

$$
\mathcal{Y}=h R \mathcal{X}+n
$$

where $h$ is channel gain and $R$ is receiver responsivity. On-off keying (OOK) modulation is applied, and $\mathcal{X} \in\{0,2 P\}$, where $P$ is the average transmitted optical power. The channel gain $h$ can be factored as

$$
h=h_{l} h_{a} h_{t r}
$$

where $h_{l}, h_{a}$ and $h_{t r}$ denote the attenuation due to atmospheric loss, scintillation and tracking error respectively.

\section{B. Atmospheric model}

The propagation loss, $h_{l}$ is defined as a deterministic function of a propagation distance $L$ by Beer-Lambert Law

$$
h_{l}(L)=e^{-C_{s c} L}
$$

where $C_{s c}$ is the scattering coefficient, selected as $0.673 \mathrm{~km}^{-1}$ $(2.92 \mathrm{~dB} / \mathrm{km})$ for a weather condition between "clear" and "light haze" [12].

For atmosphere scintillation, $h_{a}$ is a random fading coefficient due to scintillation. In order to model the distribution of $h_{a}$, an uplink Hufnagel-Valley model was applied to define the refractive-index structure parameter $C_{n}^{2}(Z)$ at different altitudes $Z$ as [11, p.481]

$$
\begin{aligned}
C_{n}^{2}(Z)= & 0.00594(s / 27)^{2}\left(10^{-5} Z\right)^{10} e^{-Z / 1000} \\
& +2.7 \times 10^{-16} e^{-Z / 1500}+A e^{-Z / 100}
\end{aligned}
$$

where $s=30 \mathrm{~m} / \mathrm{s}$ is a speed of strong wind, and $A=$ $1.7 \times 10^{-13} \mathrm{~m}^{-2 / 3}$ denotes a strong nominal ground turbulence level. Secondly, for laser wave-length $\lambda=1550 \mathrm{~nm}$, an

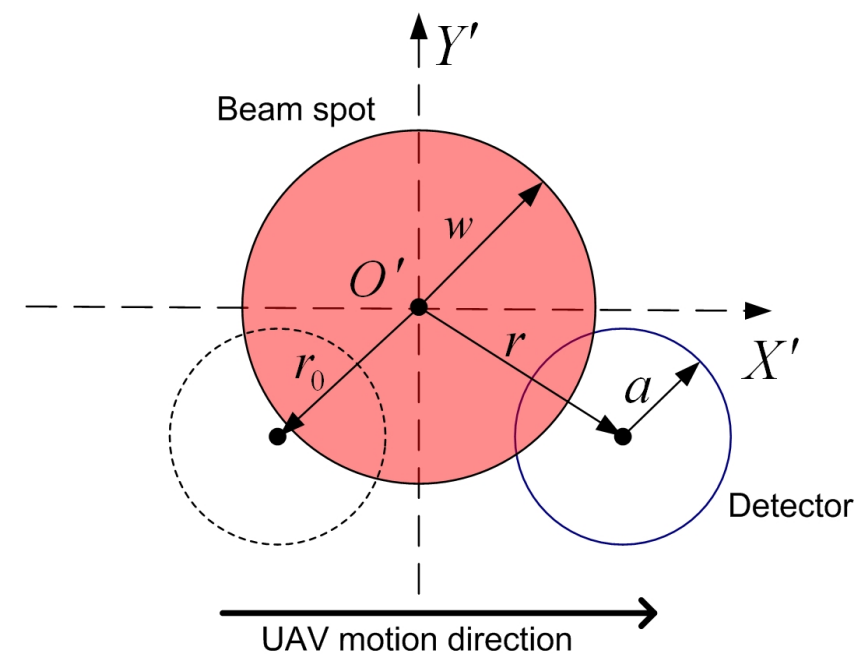

Fig. 2. Misalignment between detector and beam spot

intermediate constant $\sigma_{B u}^{2}$ is calculated from $C_{n}^{2}(Z)$ [11, p.509].

$$
\sigma_{B u}^{2}=2.25\left(\frac{2 \pi}{\lambda}\right)^{\frac{7}{6}}\left(\frac{L}{Z_{U}}\right)^{\frac{11}{6}} \int_{0}^{Z_{U}} C_{n}^{2}(Z)\left[Z-\frac{Z^{2}}{Z_{U}}\right]^{\frac{5}{6}} d Z .
$$

As is conventional, $h_{a}$ is taken to be Gamma-Gamma distributed [11]

$$
\operatorname{Pr}\left(h_{a}\right)=\frac{2(\alpha \beta)^{(\alpha+\beta) / 2}}{\Gamma(\alpha) \Gamma(\beta)}\left(h_{a}\right)^{\frac{(\alpha+\beta)}{2}-1} K_{\alpha-\beta}\left(2 \sqrt{\alpha \beta h_{a}}\right)
$$

where $K_{\alpha-\beta}(\cdot)$ is the modified Bessel function of the second kind and $\alpha, \beta$ can be expressed with $\sigma_{B u}$ as [11, p.517].

$$
\begin{aligned}
& 1 / \alpha=e^{0.49 \sigma_{B u}^{2} /\left(1+0.56 \sigma_{B u}^{12 / 5}\right)^{7 / 6}}-1, \\
& 1 / \beta=e^{0.51 \sigma_{B u}^{2} /\left(1+0.69 \sigma_{B u}^{12 / 5}\right)^{5 / 6}}-1 .
\end{aligned}
$$

\section{Tracking error}

Figure 2 is a diagram of the beam spot in the transverse plane at the receiver in a given tracking interval $T_{t r}$. Define $\vec{r}(t)$ as the instantaneous distance between centres of the beam spot and the detector in a given tracking interval, i.e., $t \in$ $\left[0, T_{t r}\right)$ as

$$
\vec{r}(t)=\vec{r}_{0}+\vec{v} t
$$

where $\vec{r}_{0}$ is the initial beam position at the start of the tracking interval (i.e., $t=0$ ) and $\vec{v}$ is a constant velocity vector with magnitude $100 \mathrm{~m} / \mathrm{s}$.

At the start of each tracking interval, $\vec{r}_{0}$ is the random initial beam position as determined by the GPS data and the gimbal error. The initial misalignments in $X^{\prime}$ and $Y^{\prime}$ are modelled as identical, independent zero-mean Gaussian distributions with variance $\sigma_{t r}^{2}$. Based on experimental data of gimbal and GPS errors, $\sigma_{t r}=3.3 \mathrm{~m}$ with $99 \%$ confidence level [3]. Thus, $\vec{r}_{0}$ is modelled as being chosen randomly and independently at the start of each tracking interval whereas the variation of $\vec{r}$ within each tracking is known given $\vec{r}_{0}$. 


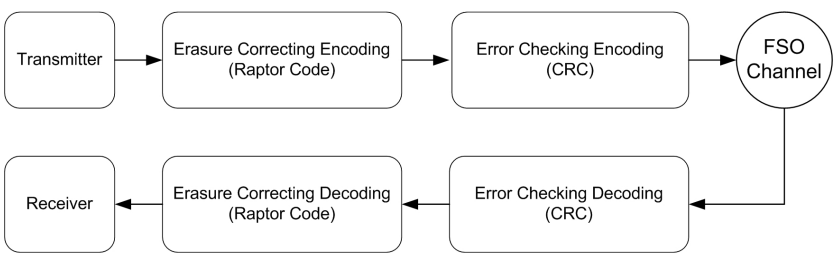

Fig. 3. Packet-Level Channel Model of ground-to-UAV FSO channel

Following [8], the intensity at the receiver due to misalignment can be estimated based on the instantaneous $\vec{r}$ in (4). Define $q=\sqrt{\pi / 2} \cdot(a / w), A_{0}=[\operatorname{erf}(q)]^{2}$, where $a$ is the radius of detector and $w$ is the laser beam waist $\left(e^{-2}\right.$ of the maximum magnitude). Also define $w_{\text {eq }}$ as the equivalent beam width, where $w_{e q}^{2}=w^{2} \sqrt{\pi} \operatorname{erf}(q) / 2 q e^{-q^{2}}$. For a given $\vec{r}$, the attenuation is [8]

$$
h_{t r}(\vec{r}) \approx A_{0} e^{-2\|\vec{r}\|^{2} / w_{\mathrm{eq}}^{2}} .
$$

It is important to note that the impact of $h_{t r}$ is often a limiting factor in the design of such mobile FSO systems. The attenuation $h_{t r}$ varies widely, even in a given $T_{t r}$, as the detector moves across the beam spot and often dominates over atmospheric scintillation. These observations will be quantified by simulations in Sec. IV.

\section{Channel model}

The ground-to-UAV FSO channel considered in this paper is modelled as a packet transmission system, and is shown in Fig. 3. A message is divided into $k$ packets each of size $1 \mathrm{kbit}$. A sequence of Raptor coded outputs are generated from these message packets, as discussed in Sec. III. A 32-bit CRC from the IEEE 802.3 standard [13] is added to each packet before transmission on the channel defined in (1) and (2). At the receiver, any corrupted packets are dropped and not used in the Raptor decoder. Since the probability of undetectable error for this CRC is $<10^{-9}$, when bit error probability $<0.5$ [13], we ignore undetected packet errors at the receiver. Finally, the entire message is decoded when sufficient packets are received. In addition, it is assumed that the transmitter continues to send packets for a given message until it receives a 1-bit feedback from the receiver to move to the next message.

\section{DESIGN OF SHORT-LENGTH RAPTOR CODES}

\section{A. Background}

Raptor codes are based on Luby transform (LT) codes [14] which are random bipartite codes where every encoded packet is a linear combination of several message packets. For a given encoded packet the number of message packets combined is termed the degree and is chosen randomly and independently for each encoded packet. Raptor codes differ from LT codes due to the use of a different degree distribution and a lowdensity parity-check (LDPC) pre-code. This LDPC pre-code enlarges the effective number of message packets from $k$ to $\hat{k}$, as shown in Fig. 4.

Define the degree distribution polynomial of a Raptor code as $\Omega(x)=\sum_{i=1}^{\hat{k}} \Omega_{i} x^{i}$, where item $x^{i}$ represents a degree $i$,

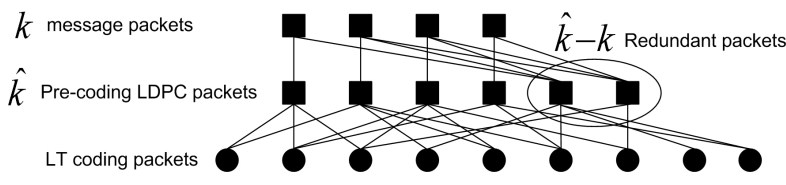

Fig. 4. Configuration of Raptor codes

and $\Omega_{i}$ is the probability mass of degree $i$. The decoding algorithm of both LT and Raptor codes is a simplified belief propagation (BP) algorithm. This algorithm starts from a degree one packet, using it to decode other packets with larger degrees. Decoded packets are subtracted from all larger degree packets which involve it. All received packets are stored in a buffer and the decoding process continues searching and subtracting until the $k$ message packets are recovered.

The complexity in decoding a Raptor code has been shown to be $O(k \log 1 / \varepsilon)$ on average, where $\varepsilon>0$ is a design code overhead and typically set at a couple percent [5]. It is important to notice that the line rate of FSO links are typically in excess of $1 \mathrm{Gbps}$ and in this application the decoder operates in the UAV which has limited processing and energy storage. Thus, in this work to limit both latency and decoding complexity we consider short-length Raptor codes of $k \leq 1024$.

The rate of a Raptor code is in fact random. Define the number of received packets necessary for successful decoding as $m$. An important parameter is the expected overhead of the Raptor code, $\varepsilon_{m}$, which is defined as

$$
\langle m\rangle=\left(1+\varepsilon_{m}\right) k
$$

where $\langle\cdot\rangle$ denotes expected value. Here, $\varepsilon_{m}$ is the statistical overhead, which is different from the design overhead $\varepsilon$. It is apparent that a smaller $\varepsilon_{m}$ implies a higher rate. Previous work on Raptor codes [5] has demonstrated that reducing $k$ incurs an increase in $\varepsilon_{m}$, i.e., a reduction in rate. In the Sec. III-C, we describe a search technique for a degree distribution to miminize $\varepsilon_{m}$ for short-length Raptor codes.

\section{B. Pre-Coding $L D P C$}

To simplify the encoder and decoder a systematic rightregular LDPC pre-code is adopted where each check has fixed degree $D$. The $D$ message packets involved at each check are chosen uniformly and independently. To each message $\hat{k}-k=$ $0.02 k$ redundant LDPC packets are added for $k=64$ to 1024 , and a single packet is added for $k=16,32$. The degree, $D$, of each redundant packet for different $k$ is shown in Tbl. I. The degree was initially set to $\log _{2} k$, which is the average degree of the LT code, and increased enough to minimize $\varepsilon_{m}$ after simulation. Note, this heuristic optimization is typical in the design of such codes [5].

\section{Design of degree distribution}

The input ripple is defined as the set of message packets decoded up to a particular step [5]. Clearly, the goal is to ensure that the input ripple as large as possible throughout the decoding process. In [5], the author gives a heuristic proposal: 
TABLE I

DEGREE OF LDPC PRE-CODE AND $c$ FOR DIFFERENT $k$

\begin{tabular}{|c|c|c|c|c|c|c|c|}
\hline$k$ & 16 & 32 & 64 & 128 & 256 & 512 & 1024 \\
\hline \hline$D$ & 9 & 16 & 19 & 27 & 30 & 33 & 44 \\
\hline$c$ & 0.04 & 0.05 & 0.05 & 0.07 & 0.08 & 0.08 & 0.09 \\
\hline
\end{tabular}

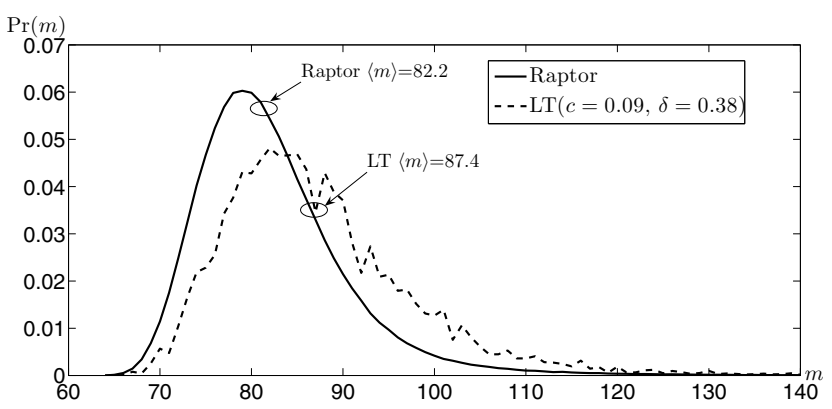

Fig. 5. $\operatorname{Pr}(m)$ of Raptor code and LT code for $k=64$

for $x \in[0,1-\delta]$, where $\delta$ is the fraction of message packets not decoded, keep the ripple size $\geq c \sqrt{(1-x) \hat{k}}$, where $c$ is a small constant. Under this proposal it was shown that $\Omega(x)$ should satisfy

$$
\Omega^{\prime}(x) \geq \frac{-\ln \left(1-x-c \sqrt{\frac{1-x}{\hat{k}}}\right)}{1+\varepsilon}, x \in[0,1-\delta]
$$

where $\Omega^{\prime}(x)$ is the derivative of $\Omega(x)$ [5].

As in [5], (7) is verified by discretizing the interval $[0,1-\delta]$ in steps of 0.001. Since there are an infinite number of possible $\Omega(x)$ which satisfy (7), the design of $\Omega(x)$ for short $k$ is based on the degree distribution for a very long Raptor code of $k=$ 64536 [5]

$$
\begin{aligned}
& \Omega^{*}(x)=0.007969 x+0.49357 x^{2}+0.16622 x^{3}+0.072646 x^{4} \\
& +0.082558 x^{5}+0.056058 x^{8}+0.037229 x^{9}+0.05559 x^{19} \\
& +0.025023 x^{65}+0.003135 x^{66} .
\end{aligned}
$$

Since there is no analytical design method for $\Omega(x)$, we present a heuristic technique for $k=16$ to 1024 . A typical value $\varepsilon=0.05$ is selected as in [5]. To verify (7), $c<\delta \sqrt{\hat{k}}$ and $\delta=0.01$ is set as in [5]. The value of $c$ must be carefully chosen to ensure (7) is satisfied. Table I shows the values of $c$ chosen after extensive simulation. To find a suitable $\Omega(x)$, $\Omega^{*}(x)$ is modified stepwise until (7) is satisfied .

Step 1: Truncate the large degree items in $\Omega^{*}(x)$ larger than $k / 4$. For example, $x^{5}$ to $x^{66}$ terms are removed for $k=16$. From [5], $\Omega(x)$ need not have packets of every degree. For example, Fig.5 shows that a truncated Raptor code with lower $\langle m\rangle$ than a comparable LT code which has all possible degree packets. Heuristically, adding large degree packets also increases $d_{\text {avg }}$ significantly.

Step 2: Add $x^{11}, x^{13}, x^{16}$ and $x^{25}$ to $\Omega(x)$ for $k=64$, 128 and 256. Larger degree packets are used to ensure that all message packets are covered. However, for the small $k$ considered, the large degrees in $\Omega^{*}(x)$ are excessive. So, we
TABLE II

DESIGN RESULTS FOR $\Omega(x)$

\begin{tabular}{|c|c|c|c|c|c|c|c|}
\hline$k$ & 16 & 32 & 64 & 128 & 256 & 512 & 1024 \\
\hline \hline$\Omega_{1}$ & 0.18 & 0.11 & 0.1 & 0.06 & 0.04 & 0.025 & 0.015 \\
\hline$\Omega_{2}$ & 0.52 & 0.5 & 0.5 & 0.495 & 0.495 & 0.495 & 0.495 \\
\hline$\Omega_{3}$ & 0.1 & 0.13 & 0.11 & 0.16 & 0.167 & 0.167 & 0.167 \\
\hline$\Omega_{4}$ & 0.2 & & 0.08 & 0.08 & 0.08 & 0.082 & 0.082 \\
\hline$\Omega_{5}$ & & 0.26 & 0.042 & 0.05 & 0.07 & 0.071 & 0.071 \\
\hline$\Omega_{8}$ & & & & 0.037 & 0.039 & 0.05 & 0.049 \\
\hline$\Omega_{9}$ & & & 0.045 & 0.02 & 0.025 & 0.044 & 0.048 \\
\hline$\Omega_{11}$ & & & 0.06 & & & & \\
\hline$\Omega_{13}$ & & & 0.063 & & & & \\
\hline$\Omega_{16}$ & & & & 0.04 & & & \\
\hline$\Omega_{19}$ & & & & 0.058 & 0.035 & 0.043 & 0.05 \\
\hline$\Omega_{25}$ & & & & & 0.049 & & \\
\hline$\Omega_{66}$ & & & & & & 0.023 & 0.023 \\
\hline
\end{tabular}

add these moderate degree terms as a trade-off between $x^{9}$ and $x^{66}$ terms.

Step 3: Increase $\Omega_{i}$ for small degrees significantly, especially for degree one. For example, for $k=16$, increase $\Omega_{1}$ from 0.007969 to 0.18 . After $\Omega^{*}(x)$ is truncated in step $1, \Omega_{i}$ of large degree terms is shifted to smaller degrees. If $\Omega_{1}$ increases, more degree one packets are released in the early stage of decoding. In addition, this shifting reduces $d_{\mathrm{avg}}$, the average decoding cost. However, if $\Omega_{1}$ is too large, the encoded packets cannot cover enough message packets and decoding can stall. Therefore, $\Omega_{1}$ is increased and checked via simulation to ensure good operation.

After the heuristic steps above, an initial $\Omega(x)$ is obtained for each $k$. However, this design is often not robust with large $d_{\text {avg }}$ and $\varepsilon_{m}$. The $\Omega(x)$ found after the steps is taken as the initial value of a code search with the objective of reducing both $\varepsilon_{m}$ and $d_{\text {avg. }}$. A simulation-based technique is used to refine this initial $\Omega(x)$. For each $k$ and choice of $\Omega(x)$ many simulations are performed to estimate the distribution of $m$. An example, the distribution of $m$ is shown in Fig.5 for $k=64$ for $10^{8}$ iterations. Heuristically, if $\varepsilon_{m}$ is large from simulation, $\Omega(x)$ is changed by shifting $\Omega_{i}$ from large degree to small degree. This search procedure continues in this fashion until $\varepsilon_{m}$ cannot be reduced any further. Table II presents the resulting degree distributions.

\section{Results of code design}

Figure 6 has the $\varepsilon_{m}$ and $d_{\text {avg }}$ for the codes defined in Tbl. II. Notice that $\varepsilon_{m}$ decreases with increasing $k$. When $k=1024, \varepsilon_{m}$ decreases to 0.126 . However, $d_{\text {avg }}$ increases with $k$. For $k=1024, d_{\text {avg }}=6.32$, which can be considered as a moderate decoding cost. Compared to $k=64536$ code using $\Omega^{*}(x)$ from (8) [5], coding overhead, $\varepsilon_{m}$, for $k=1024$ is $8 \%$ larger than for $k=64536$, however, $d_{\text {avg }}$ is approximately $37 \%$ less. This is a significant trade-off between a slight increase in coding overhead $\varepsilon_{m}$ and a large decrease in decoding complexity, i.e., $d_{\text {avg }}$.

\section{Simulation Results ON FSO CHANNEL}

In this section, the Raptor codes of Sec. III are applied on the mobile FSO channel of Sec. II. The channel state is 


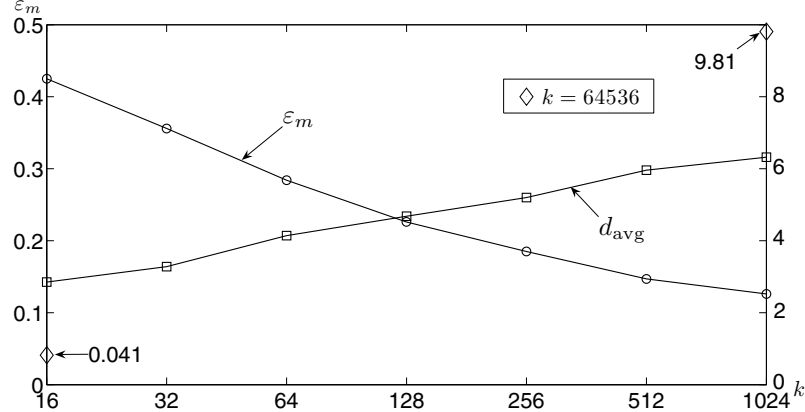

Fig. 6. Overhead, $\varepsilon_{m}$, and Average degree, $d_{\mathrm{avg}}$, of Raptor codes

randomly selected according to $h_{a}$ and $h_{t}$ and Gaussian noise is added to each packet. The received packets that fail the CRC are discarded. As in Sec. II-D, a one bit feedback is assumed to tell the transmitter to proceed to the next $k$-packet message.

The performance of the system with Raptor codes is compared to uncoded automatic repeat-request (ARQ) algorithm. Notice that ARQ requires that the transmitter must wait for feedback from the receiver for every packet. For a fair comparison, the total delay of packet propagation and feedback signal is considered. It is assumed that the 1-bit feedback for ARQ is perfect. Thus, the total delay is $2 L / C=11.5 \mu \mathrm{s}$, where $C$ is the speed of light. Note that this delay is much larger than the packet transmission duration $1 \mu$ s and severely limits rates using ARQ.

The average data rates when using an OOK, 1 Gbps transmitter are simulated. The average transmit power, $P$, is varied from $10-300 \mathrm{~mW}(10-25 \mathrm{dBm})$ and the receiver noise standard deviation $\sigma_{n}=10^{-7}$ A from [8]. The UAV speed is set to $100 \mathrm{~m} / \mathrm{s}(360 \mathrm{~km} / \mathrm{h})$, and GPS updates happen every $50 \mathrm{~ms}$ for a total simulation duration of $100 \mathrm{~s}$ [3]. Other channel parameters are selected as mentioned in Sec II. Raptor codes are compared to the the capacity of the equivalent packet erasure channel, and ARQ in Fig. 7.

The average data rate increases with $P$ leaving a small gap to the erasure capacity. With increasing $k$, the average data rate also increases. It is obvious that the incremental performance improvement from $k=512$ to 1024 is much smaller than that from $k=16$ to 32 . So, it is suggested that a practical choice is $k=64$ which provides and average rate of $560 \mathrm{Mbps}$ for $P=20 \mathrm{dBm}$ and $d_{\mathrm{avg}}=4.14$ average operations per packet.

Notice that the maximum rate using ARQ is significantly smaller than the Raptor coded system. The short-length Raptor codes designed here improve the data rate from $60 \mathrm{Mbps}$ to $560 \mathrm{Mbps}$. In addition, 4.14 binary operations per packet are required which is acceptable for a small decoding latency, especially for this high rate link. The rate improvement of short-length Raptor codes over ARQ requires a small hardware cost and storage at both encoder and decoder.

\section{CONCLusions}

In this paper, we design short-length Raptor codes for a ground-to-UAV mobile FSO channel. These codes are inde-

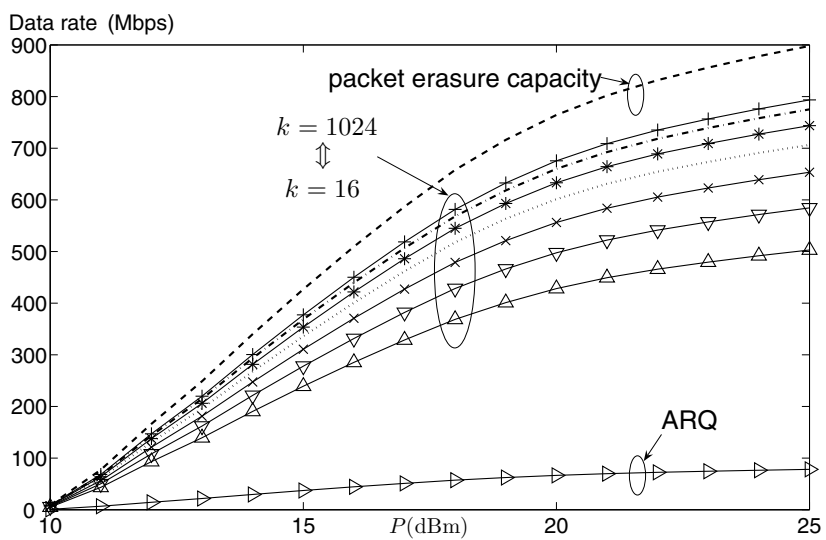

Fig. 7. Average data rate versus transmitted power on mobile FSO channel

pendent of channel misalignment caused by tracking error and atmosphere scintillation. Since the designed codes are independent of the channel state and have low complexity they are appropriate for a host of mobile FSO applications. Although the misalignment is large, for code length $k=64$, the Raptor-coded mobile FSO channel offers average rates of $560 \mathrm{Mbps}$ and a low decoding cost of $d_{\mathrm{avg}}=4.14$ per packet using a $1 \mathrm{Gbps}$ transmitter and $20 \mathrm{dBm}$ transmit power.

\section{REFERENCES}

[1] M.C. Jeong, J.S. Lee, S.Y. Kim, S.W. Namgung, J.H. Lee, M.Y. Cho, S.W. Huh and J.S. Lee, " $8 \times 10 \mathrm{~Gb} / \mathrm{s}$ terrestrial optical free space transmission over $3.4 \mathrm{~km}$ using an optical repeater," Proc. IEEE Opt. Fiber Commun. Conf. Exhib., pp. 405-407, 2002.

[2] V. Gadwal and S. Hammel, "Free-space optical communication links in a marine environment," Proc. SPIE, vol. 6304, pp. 1-11, 2006.

[3] T. I. King, H. H. Refai, J. J. Sluss Jr. and Y. Lee, "Control system analysis for ground/air-to-air laser communications using simulation", Proc. IEEE 24th Digital Avionics Syst. Conf., pp. 1.C.3-1-1.C.3-7, 2005.

[4] A. Harris, J. J. Sluss Jr. and H. H. Refai, "Alignment and tracking of a Free-Space Optical communication link to a UAV", Proc. IEEE 24th Digital Avionics Syst. Conf., pp. 1.C.2-1-1.C.2-9, 2005.

[5] A. Shokrollahi, "Raptor codes," IEEE Trans. Inform. Theory, vol. 52, no. 6, pp. 2551-2567, June 2006.

[6] A. Biswas and S. Piazzolla, "Deep-Space Optical Communications Downlink Budget from Mars: System Parameters," Interplanetary Network Progress Report, Jet Propulsion Laboratory, 2003.

[7] C. Anderson, "Transformational Communications," Rev.5, MILSATCOM Joint Program Office, 2002. [online], http://sunset.usc.edu/GSAW/gsaw2002/s8/canderson.pdf.

[8] A. A. Farid and S. Hranilovic, "Outage Capacity Optimization for FreeSpace Optical Links With Pointing Errors," IEEE/OSA J. Lightwave Tech., vol. 25, no. 7, pp. 1702-1710, Jul. 2007.

[9] Z. Wu, H. Kumar and A. Davari, "Performance evaluation of OFDM transmission in UAV wireless communication," Proc. 37th Southeastern Symp. on Syst. Avionics, pp. 6-10, 2005.

[10] R. Palanki and J. S. Yedidia, "Rateless codes on noisy channels," Proc. IEEE Int. Symp. Inform. Theory, pp. 38, 2004.

[11] L. C. Andrews and R. L. Phillips, "Laser Beam Propagation through Random Media," second edition, SPIE press, 2005.

[12] E. J. McCarteny, "Optics of the Atmosphere," Wiley press, 1976.

[13] T. Fujiwara, T. Kasami and S. Lin, "Error detecting capabilities of the shortened Hamming codes adopted for error detection in IEEE standard 802.3," IEEE Trans. Commun., vol. 37, no. 9, pp. 986-989, Sep. 1989.

[14] M. Luby, "LT codes," Proc. ACM Symp. Found. Comput. Sci., pp. 271$280,2002$. 